\title{
Levantamento da rede de atenção aos usuários de drogas: Um estudo exploratório
}

Pedro Henrique Antunes da Costa. Faculdade Machado Sobrinho.

Amata Xavier Medeiros. Universidade Federal de Juiz de Fora.

Bárbara Pereira Loures. Universidade Federal de Juiz de Fora.

Wanderson Maurício Duarte Silva. Universidade Federal de Juiz de Fora.

Telmo Mota Ronzani. Universidade Federal de Juiz de Fora.

Fernando Antonio Basile Colugnati. Universidade Federal de Juiz de Fora.

\section{Resumo}

O presente estudo realizou um levantamento e análise da rede de atenção aos usuários de drogas de Juiz de Fora, Minas Gerais. Os procedimentos utilizados foram: 36 entrevistas e coleta de informações sobre os serviços junto a gestores/profissionais e em bases de dados. Foram identificados 184 serviços assistenciais, sendo 114 do setor saúde, 16 da assistência social e 54 recursos comunitários. Apesar da heterogeneidade de dispositivos e abordagens, existe uma hegemonia de oferta de serviços não governamentais na assistência aos usuários de drogas. As seguintes conclusões são feitas: necessidade de ampliação da rede de atenção básica; implantação de mais Centros de Atenção Psicossocial em álcool e drogas; expansão da rede atenção psicossocial; reestruturação da atenção a mulheres, crianças e adolescentes; ampliação da rede de urgência e emergência; e maior distribuição dos serviços, principalmente os governamentais especializados.

Palavras-chave: serviços de saúde pública; droga (uso); droga (abuso); saúde mental; políticas públicas.

\begin{abstract}
Survey of the care network for drug users: An exploratory study. The study surveyed and analyzed the care network for drug users of Juiz de Fora, Minas Gerais. The proceedings were: 36 interviews and data collection about services with managers/ professionals and on databases. One hundred eighty four care services were identified, with 114 of the health sector, 16 from social care and 54 community resources. Despite the heterogeneity of devices and approaches, there was a hegemony of non-governmental service provision in the care of drug users. The following conclusions are made: need of expanding primary care network; deployment of more Psychosocial Care Centers for alcohol and drugs; expansion of psychosocial care network; rearrangement of care to women, children and adolescents; expansion of the emergency and urgent care network; and greater distribution of services, especially the governmental ones that are specialized for drug users.
\end{abstract}

Keywords: public health services; drug usage; drug abuse; mental health; public policies.

\section{Resumen}

Survey de la red de atención a los usuarios de drogas: Un estudio exploratorio. Este estudio tuvo como objetivo analizar la red de atención a los usuarios de drogas de Juiz de Fora, Minas Gerais. Los procedimientos utilizados fueron: 36 entrevistas y recopilación de informaciones con los gerentes/profesionales y em las bases de datos. Fueran identificados 184 servicios de atención, siendo 114 de la salud, 16 de la asistencia social y 54 recursos comunitarios. A pesar de la heterogeneidad de los dispositivos y enfoques, hay una hegemonía en la prestación de servicios no gubernamentales a los usuarios de drogas. Las siguientes conclusiones son realizadas: necesidad de expansión de la atención primaria; más Centros de Atención Psicosocial - alcohol y drogas; expansión de la red de atención psicosocial; reestructuración de la atención a las mujeres, niños y adolescentes; ampliación de la red de atención de emergencia y urgencia; y una mayor distribución de los servicios, especialmente los especializado gubernamentales. Palabras clave: servicios de salud pública; drogas (uso); abuso de drogas; salud mental; políticas públicas. 
Atualmente no Brasil, os sistemas de cuidado aos usuários de álcool e outras drogas são estruturados através de redes de atenção, sendo essas as respostas sociais das políticas públicas para atender as necessidades dos usuários de substâncias psicoativas (Ministério da Saúde [MS], 2004; Secretaria Nacional de Políticas sobre Drogas [SENAD], 2005). Essas redes são construções recentes visando minimizar uma lacuna assistencial originada pela negligência à temática atrelada a abordagens criminalizatórias (Alves, 2009). Pressupõem uma tentativa de organização de serviços e conformação de uma rede intersetorial e integrada, em detrimento de iniciativas isoladas e/ou sistemas fragmentados (MS, 2004; SENAD, 2005).

Nesse sentido, são necessários estudos que visem entender a realidade assistencial aos usuários de drogas no Brasil. Relata-se a existência de levantamentos prévios de serviços assistenciais aos usuários de drogas em Florianópolis (Spohr, Leitão, \& Schneider, 2006), no Espírito Santo (Siqueira, Barbosa, Laranjeira, \& Hopkins, 2007), na região Centro-oeste (Morais, 2008), além de um levantamento nacional (Carvalho, 2007). Estes estudos enfocam nos serviços especializados, como os Centros de Atenção Psicossocial para álcool e drogas (CAPSad), clínicas/comunidades terapêuticas (CTs) e grupos de ajuda mútua (GAMs). Contudo, devido à complexidade da problemática, perpassada por novas reformulações políticas no Sistema Único de Saúde (SUS), como a Reforma Psiquiátrica (RP), a implantação da Rede de Atenção Psicossocial (RAPS) (Portaria ${ }^{\circ}$ 3.088 , de 23 de dezembro de 2011), e a própria configuração dos sistemas assistenciais aos usuários de drogas, entende-se que esta rede vai além dos serviços especializados e do setor saúde (Rush, 2010).

As próprias políticas da área, a Política Nacional sobre Drogas (PNAD) da Secretaria Nacional de Políticas sobre Drogas (SENAD, 2005), e a Política de Atenção Integral aos Usuários de Álcool e outras Drogas (PAIUAD) (MS, 2004), postulam que uma abordagem por redes intersetoriais integradas possibilita abordar holisticamente o fenômeno, considerando o continuum de cuidados, com prevenção, diversas modalidades de tratamento e reinserção social. Dessa forma, entende-se a rede de atenção aos usuários de drogas como a conjunção de serviços e modalidades de atenção do SUS, especificamente das redes de atenção à saúde (RAS), e RAPS, da rede do Sistema Único de Assistência Social (SUAS) e com a complementaridade dos recursos assistenciais comunitários, que são dispositivos não governamentais de base comunitária, como associações, entidades socioassistenciais e GAMs.

Portanto, em consonância com Costa, Mota Paiva e Ronzani (2015), essa carência de estudos que incorporam as recentes modificações da organização assistencial aos usuários de drogas no Brasil gera uma lacuna de conhecimento. Tomando como base essa noção ampliada de rede, o presente estudo realizou um levantamento e análise da rede de atenção aos usuários de drogas de um município de médio porte brasileiro, levantando implicações para as políticas públicas na área.

\section{Materiais e métodos}

Estudo transversal, com levantamento dos serviços que constituem a rede de atenção aos usuários de drogas do município de Juiz de Fora. A coleta dos dados foi realizada de agosto de 2013 a fevereiro de 2014 . O levantamento se justifica pela necessidade de compreensão da rede como um todo, tendo, assim, uma visão panorâmica do cenário assistencial aos usuários de drogas no município, fator esse que pode ser minimizado ou distorcido por análises que focalizem em dispositivos específicos, descolando-os do contexto no qual se inserem e conformam.

Juiz de Fora situa-se na região da Zona da Mata, Minas Gerais, Brasil, com área de $1.436 \mathrm{~km}^{2}$, densidade demográfica de 359,59 hab. $/ \mathrm{km}^{2}$ e grau de urbanização de 99,2. A população estimada é de 545.942 habitantes, com Índice de Desenvolvimento Humano (IDH) de 0,778 e Produto Interno Bruto (PIB) de R\$7.180.719,00 (Instituto Brasileiro de Geografia e Estatística [IBGE], 2014).

Conforme mencionado, os serviços mapeados poderiam ser governamentais ou não governamentais, constituintes do setor saúde (RAS, RAPS), SUAS ou recursos comunitários localizados no município. As abordagens de tratamento se dividiam nos seguintes tipos: 1) psicossocial, que é uma proposta de serviços abertos, sem internação, englobando aspectos psicológicos e sociais, envolvendo equipe multidisciplinar, o usuário e seu contexto de vida; 2) ambulatorial, com abordagens clínicas psicoterápicas, médicas e/ou farmacológicas, tratando clinicamente o usuário de drogas sem afastá-lo do seu cotidiano, sendo frequentemente englobada pelas abordagens psicossociais; 3) ajuda mútua, através de grupos de apoio de usuários, ex-usuários ou familiares com a abordagem; 4) internação, podendo ser para desintoxicação, reservada aos 
casos que demandam cuidados intensivos e internação psiquiátrica; e 5) comunidade terapêutica, abordagem de caráter residencial de médio a longo prazo, com elementos terapêuticos como disciplina, trabalho, espiritualidade, dentre outros (Brasil; 2004; 2005; 2011; Rush, 2010). Serviços de outros municípios, mesmo recebendo usuários de Juiz de Fora, foram desconsiderados por não fazerem parte da rede local.

O mapeamento contou inicialmente com a colaboração de secretarias gestoras municipais, através do Comitê Intergestor de Políticas sobre Drogas, além da utilização de um catálogo contendo instituições socioassistenciais do município. Nesse levantamento preliminar foram identificados 121 serviços assistenciais a usuários de drogas. Foram realizadas buscas em bases do SUS (DATASUS), SUAS, SENAD e com os Conselhos Municipais de Assistência Social (CMAS) e de Políticas Integradas sobre Drogas (COMPID), levantando mais 46 serviços. A partir dessas duas etapas, utilizou-se a estratégia de recrutamento bola de neve (snowball) (Biernacki \& Waldford, 1981), identificando e entrevistando profissionais dos serviços para que indicassem serviços ainda não mapeados, ampliando o levantamento. Surgindo novas instituições, profissionais destes serviços eram entrevistados para a identificação de dispositivos ainda não mapeados até que não fossem mencionadas novas instituições. A partir da bola de neve, foram detectados 24 novos dispositivos, mas sete serviços previamente identificados foram excluídos por não estarem mais em funcionamento ou deixarem de atender pessoas com problemas decorrentes do uso de drogas. Ao todo, para o levantamento da rede, foram realizadas 36 entrevistas por telefone e presenciais, com profissionais dos serviços e atores da gestão municipal.

Por fim, todas as instituições mapeadas foram contatadas, coletando-se as seguintes informações: endereço, região administrativa, tipo de tratamento ofertado, público alvo, natureza, financiamento do serviço e setores (ver Figura 1). Os dados coletados foram tabulados no Excel, com extração de análises descritivas.

O estudo foi aprovado pelo Comitê de Ética, parecer $n^{\circ}$ 568.878. Apesar de estar vinculado à universidade, os resultados foram disseminados e discutidos junto a dispositivos da própria rede e representantes da gestão de vários setores, como a saúde, assistência social, dentre outros. Cabe ressaltar somente que, apesar de importante, o levantamento e caracterização da rede constituem uma etapa de compreensão da rede, entendendo que essa rede é moldada, por exemplo, pelas relações que os dispositivos estabelecem entre si.

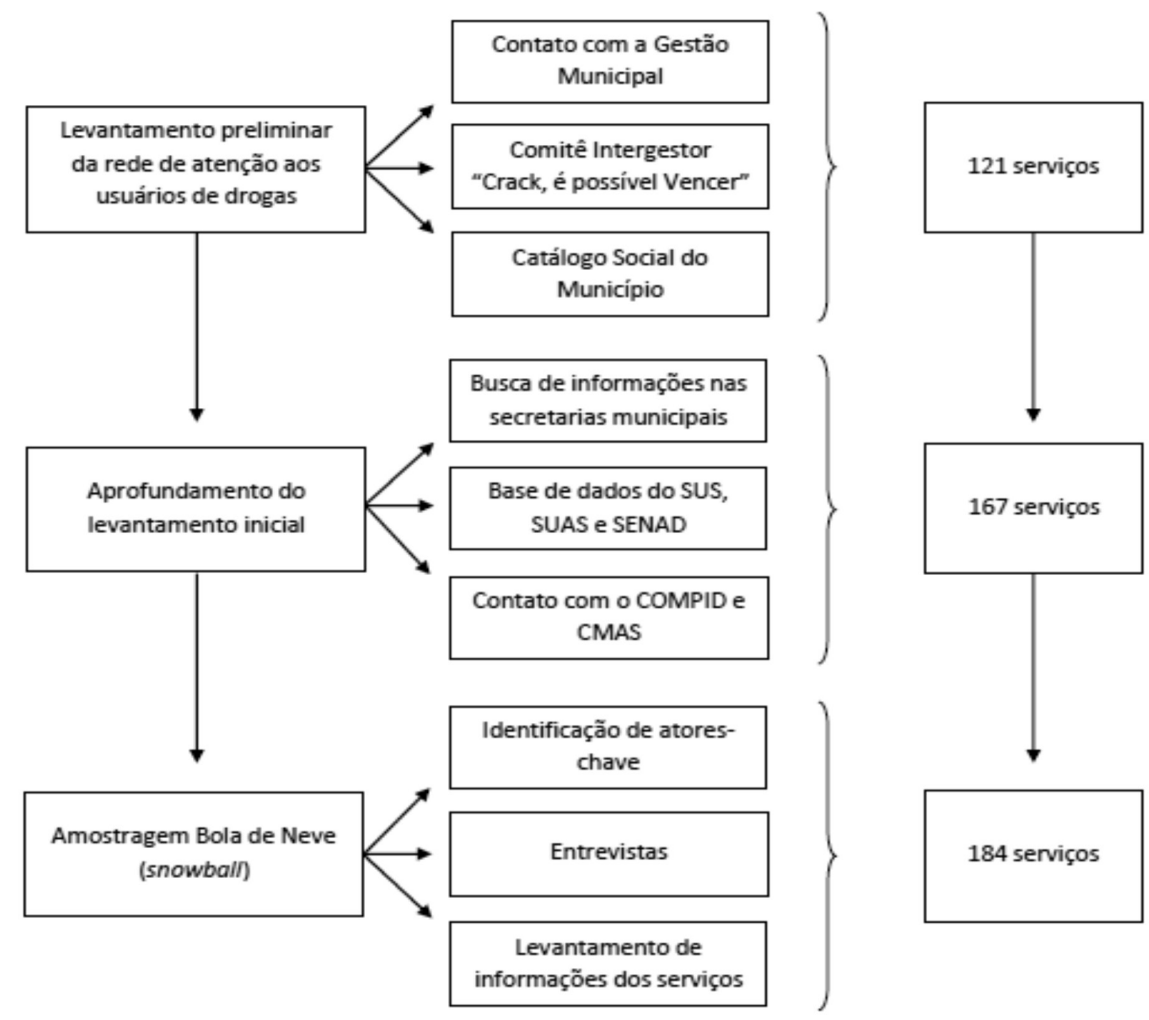

Figura 1. Processo de Levantamento da Rede de Atenção aos Usuários de Drogas 


\section{Resultados}

Foram mapeados 184 serviços/instituições que ofertavam assistência a usuários de drogas em Juiz de Fora, compondo a rede de atenção aos usuários de drogas. Destes, 114 serviços originam-se do setor saúde (RAS e RAPS), 16 da Rede SUAS e 54 serviços caracterizavam-se como recursos comunitários.

De acordo com a tabela 1, cerca da metade dos dispositivos são de natureza governamental. Entretanto, considerando somente os dispositivos específicos ao tratamento de usuário de drogas, tem-se que somente seis serviços são governamentais: o Centro de Atenção Psicossocial para álcool e drogas (CAPSad), que é o principal serviço especializado na assistência a usuários de drogas, com cuidado territorializado e em regime de tratamento intensivo, semi-intensivo e não intensivo; dois hospitais com leitos específicos para internação de curto prazo aos usuários de drogas; um ambulatório para tabagistas e outro para usuários de drogas no hospital universitário; e um hospital do sistema prisional específico para atender internos como problemas decorrentes do uso de drogas. O restante dos serviços exclusivos para usuários de drogas são os 44 GAMs, com Alcoólicos Anônimos (AA), Narcóticos Anônimos (NA), Grupos Familiares de Alcoólicos Anônimos (AL-ANON) e de Narcóticos Anônimos (NAR-ANON) e Amor Exigente para familiares de dependentes de álcool e outras drogas, além de 20 clínicas/CTs. Portanto, 114 serviços são generalistas, ou seja, não-especializados e dos 70 serviços especializados na atenção aos usuários de drogas, $64(91,4 \%)$ são de natureza não governamental e apenas seis $(8,6 \%)$ governamentais.

Com relação às regiões dos serviços, a figura 2 e tabela 2 demonstram uma maior concentração na região central, com $24 \%$ dos serviços localizados nesta região. A região com menos serviços é a oeste com 10 serviços $(5,4 \%)$. A média de serviços por região foi de $23,0(d p=9,8)$ e a média de serviços governamentais foi de $11,9(d p=4,8)$. As regiões urbanas com o menor número de dispositivos governamentais foram a oeste (com quatro), sul (sete) e nordeste (oito). As regiões central e norte (com 16 cada), leste (13) e sudeste (12) tiveram as maiores quantidades de serviços governamentais.

Os seis serviços de natureza governamental específicos para atender usuários de drogas situam-se nas regiões central (CAPSad e dois ambulatórios), nordeste (dois hospitais gerais com leitos para usuários de drogas) e leste (o hospital do sistema prisional para usuários de drogas). Os GAMs estão localizados em todas as regiões do município, com 18 grupos na região central, 10 na região norte, oito na leste, três nas regiões sudeste e oeste cada e dois nas regiões sul e nordeste. As CTs estão mais concentradas nas regiões sul e central, com sete e cinco dispositivos em cada região, respectivamente, além de dois serviços nas regiões leste, nordeste e oeste e um nas regiões sudeste e rural.

Tabela 1. Serviços Assistenciais a Usuários de Drogas no Município de Acordo com Sua Natureza Governamental

\begin{tabular}{|c|c|c|}
\hline Tipos de serviço de acordo com sua natureza & $\mathbf{N}$ & $\%$ \\
\hline Governamentais & 93 & 50,5 \\
\hline Unidades Básicas de Saúde (UBS) Urbanas & 48 & 26,1 \\
\hline Unidades Básicas de Saúde (UBS) Rurais & 19 & 10,4 \\
\hline Centros de Atenção Psicossocial (CAPS) gerais & 3 & 1,6 \\
\hline $\begin{array}{l}\text { Centros de Atenção Psicossocial para álcool e drogas } \\
\text { (CAPSad) }\end{array}$ & 1 & 0,5 \\
\hline Centros de Atenção Psicossocial infantil (CAPS i) & 1 & 0,5 \\
\hline $\begin{array}{l}\text { Ambulatórios em Saúde Mental e Álcool e outras } \\
\text { Drogas }\end{array}$ & 2 & 1,1 \\
\hline $\begin{array}{l}\text { Hospitais com leitos, ambulatórios ou enfermarias } \\
\text { para álcool e outras drogas }\end{array}$ & 5 & 2,7 \\
\hline Serviço de Atendimento Móvel de Urgência (SAMU) & 1 & 0,5 \\
\hline Centros de Referência da Assistência Social (CRAS) & 9 & 4,9 \\
\hline $\begin{array}{l}\text { Centros de Referência Especializados da Assistência } \\
\text { Social (CREAS) }\end{array}$ & 4 & 2,2 \\
\hline $\begin{array}{l}\text { Governamentais gerenciados por entidades não go- } \\
\text { vernamentais }\end{array}$ & 13 & 7,1 \\
\hline Equipes de Consultório na Rua & 1 & 0,5 \\
\hline Residências Terapêuticas & 12 & 6,6 \\
\hline Não governamentais com fins lucrativos & 11 & 6,0 \\
\hline $\begin{array}{l}\text { Hospitais com leitos, ambulatórios ou enfermarias } \\
\text { para álcool e outras drogas (Hospital Psiquiátrico) }\end{array}$ & 1 & 0,5 \\
\hline Clínicas e Comunidades Terapêuticas & 10 & 5,5 \\
\hline Não governamentais sem fins lucrativos & 67 & 36,4 \\
\hline Entidades Socioassistenciais1 & 9 & 4,9 \\
\hline Clínicas e Comunidades Terapêuticas & 10 & 5,5 \\
\hline Grupos de Ajuda Mútua & 44 & 23,9 \\
\hline Centros de Convivência & 1 & 0,5 \\
\hline Serviços de Acolhimento Institucional & 3 & 1,6 \\
\hline Total & 184 & 100,0 \\
\hline
\end{tabular}

${ }^{1}$ Entidades não governamentais de caráter assistencial que atendem usuários de drogas, dentre outras ações e públicos-alvo.

Fonte: Autores

Referente à rede de dispositivos do SUAS na atenção aos usuários de drogas, o município contava na proteção básica com nove CRAS, atendendo crianças, adolescentes e adultos de todos os sexos. $\mathrm{Na}$ 
proteção especial (média complexidade) existiam um Centro de Referência Especializado para a População em situação de rua (CentroPop) e três CREAS: um para a infância e juventude, outro específico para mulheres vítimas de violência e idosos, e o terceiro de caráter generalista. $\mathrm{Na}$ alta complexidade, foram mapeados três dispositivos de acolhimento institucional: dois funcionavam em regime de albergue para a população adulta em situação de vulnerabilidade social com vivência de rua e outro para adultos do sexo masculino. Os três serviços, apesar de não governamentais, possuíam parceria com o município por uma associação sem fins lucrativos.

Conforme demonstra a tabela 3 , apesar de 176 serviços terem como foco de assistência os usuários de drogas, os serviços governamentais e alguns serviços não governamentais também prestam assistência aos familiares. Entretanto, oito serviços priorizam a assistência aos familiares de dependentes de drogas, sendo GAMs: quatro grupos AL-ANON, três grupos NAR-ANON e um grupo do Amor Exigente.

Dos serviços exclusivos ao tratamento de mulheres, uma CT e uma entidade socioassistencial situam-se na região oeste, sendo somente a primeira especializada no atendimento a usuárias de drogas. Os serviços que atendem somente homens se dividem em 13 CTs nas regiões central, norte, nordeste, sul, sudeste e zona rural, duas entidades socioassistenciais na região central, um serviço de acolhimento institucional também na região central e um hospital geral com leitos para usuários de drogas na região nordeste. Os 165 serviços restantes prestam assistência a pessoas de ambos os sexos.

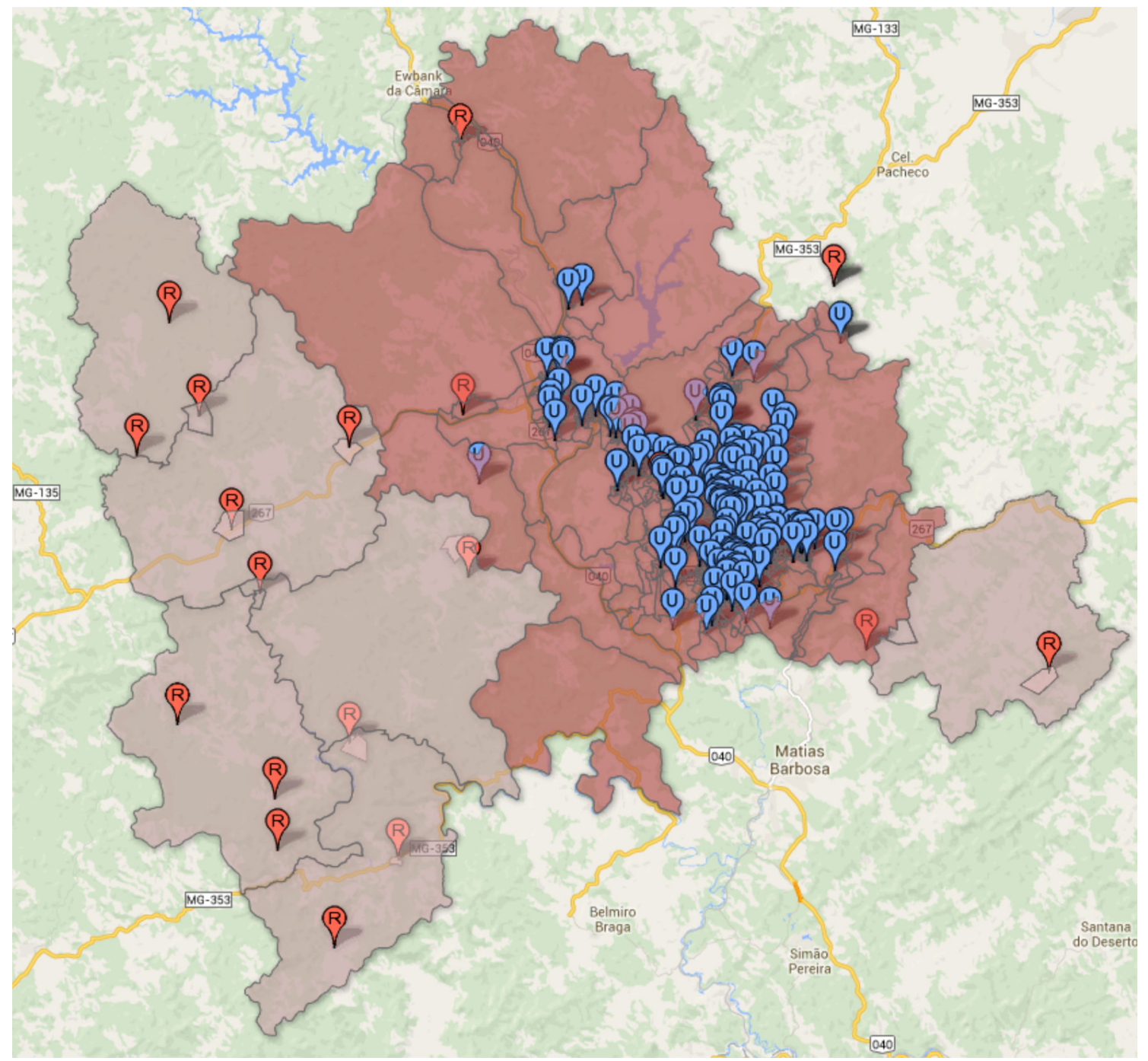

Figura 2. Distribuição dos Serviços de Acordo com Unidades Territoriais do Instituto Brasileiro de Geografia e Estatística (IBGE) 
Tabela 2. Serviços de Acordo com Suas Regiões

\begin{tabular}{|c|c|c|}
\hline Tipos de serviço de acordo com suas regiões & $\mathrm{N}$ & $\%$ \\
\hline Central & 44 & 23,9 \\
\hline Unidades Básicas de Saúde (UBS) Urbanas & 3 & 1,6 \\
\hline Centros de Atenção Psicossocial (CAPS) gerais & 2 & 1,1 \\
\hline $\begin{array}{l}\text { Centros de Atenção Psicossocial para álcool e drogas } \\
\text { (CAPSad) }\end{array}$ & 1 & 0,5 \\
\hline Centros de Atenção Psicossocial infantil (CAPS i) & 1 & 0,5 \\
\hline $\begin{array}{l}\text { Ambulatórios em Saúde Mental e Álcool e outras } \\
\text { Drogas }\end{array}$ & 2 & 1,1 \\
\hline $\begin{array}{l}\text { Hospitais com leitos, ambulatórios ou enfermarias } \\
\text { para álcool e outras drogas }\end{array}$ & 2 & 1,1 \\
\hline Serviço de Atendimento Móvel de Urgência (SAMU) & 1 & 0,5 \\
\hline Centros de Referência da Assistência Social (CRAS) & 1 & 0,5 \\
\hline $\begin{array}{l}\text { Centro de Referência Especializados da Assistência } \\
\text { Social (CREAS) }\end{array}$ & 3 & 1,6 \\
\hline Centros de Convivência & 1 & 0,5 \\
\hline Clínicas e Comunidades Terapêuticas & 5 & 2,8 \\
\hline Serviços de Acolhimento Institucional & 1 & 0,5 \\
\hline Entidades Socioassistenciais & 5 & 2,8 \\
\hline Grupos de Ajuda Mútua & 16 & 8,8 \\
\hline Leste & 23 & 12,5 \\
\hline Unidades Básicas de Saúde (UBS) Urbanas & 9 & 4,9 \\
\hline Centros de Atenção Psicossocial (CAPS) gerais & 1 & 0,5 \\
\hline $\begin{array}{l}\text { Hospitais com leitos, ambulatórios ou enfermarias } \\
\text { para álcool e outras drogas }\end{array}$ & 1 & 0,5 \\
\hline Centros de Referência da Assistência Social (CRAS) & 2 & 1,1 \\
\hline Serviços de Acolhimento Institucional & 1 & 0,5 \\
\hline Entidades Socioassistenciais & 1 & 0,5 \\
\hline Grupos de Ajuda Mútua & 8 & 4,5 \\
\hline Nordeste & 12 & 6,5 \\
\hline Unidades Básicas de Saúde (UBS) Urbanas & 5 & 2,8 \\
\hline $\begin{array}{l}\text { Hospitais com leitos, ambulatórios ou enfermarias } \\
\text { para álcool e outras drogas }\end{array}$ & 2 & 1,1 \\
\hline Clínicas e Comunidades Terapêuticas & 2 & 1,1 \\
\hline Centros de Referência da Assistência Social (CRAS) & 1 & 0,5 \\
\hline Grupos de Ajuda Mútua & 2 & 1,1 \\
\hline Norte & 28 & 15,2 \\
\hline Unidades Básicas de Saúde (UBS) Urbanas & 14 & 7,6 \\
\hline Centros de Referência da Assistência Social (CRAS) & 1 & 0,5 \\
\hline $\begin{array}{l}\text { Centro de Referência Especializados da Assistência } \\
\text { Social (CREAS) }\end{array}$ & 1 & 0,5 \\
\hline Clínicas e Comunidades Terapêuticas & 2 & 1,1 \\
\hline Grupos de Ajuda Mútua & 10 & 5,5 \\
\hline Oeste & 10 & 5,4 \\
\hline Unidades Básicas de Saúde (UBS) Urbanas & 3 & 1,6 \\
\hline Centros de Referência da Assistência Social (CRAS) & 1 & 0,5 \\
\hline Clínicas e Comunidades Terapêuticas & 2 & 1,1 \\
\hline Entidades Socioassistenciais & 1 & 0,5 \\
\hline Grupos de Ajuda Mútua & 3 & 1,6 \\
\hline Sudeste & 24 & 13,0 \\
\hline Unidades Básicas de Saúde (UBS) Urbanas & 8 & 4,5 \\
\hline Centros de Referência da Assistência Social (CRAS) & 2 & 1,1 \\
\hline Equipe de Consultório na Rua (UBS de referência) & 1 & 0,5 \\
\hline Residências Terapêuticas & 7 & 3,8 \\
\hline Serviços de Acolhimento Institucional & 1 & 0,5 \\
\hline
\end{tabular}

Tabela 2. Continuação

\begin{tabular}{lcc}
\hline Tipos de serviço de acordo com suas regiões & N & $\%$ \\
\hline Clínicas e Comunidades Terapêuticas & 1 & 0,5 \\
Grupos de Ajuda Mútua & 3 & 1,6 \\
Hospitais com leitos, ambulatórios ou enfermarias & 1 & 0,5 \\
para álcool e outras drogas (Hospital Psiquiátrico) & 23 & 12,5 \\
Sul & 6 & 3,2 \\
Unidades Básicas de Saúde (UBS) Urbanas & 1 & 0,5 \\
Centros de Referência da Assistência Social (CRAS) & 5 & 2,8 \\
Residências Terapêuticas & 7 & 3,8 \\
Clínicas e Comunidades Terapêuticas & 2 & 1,1 \\
Entidades Socioassistenciais & 2 & 1,1 \\
Grupos de Ajuda Mútua & 20 & 11,0 \\
Rural & 19 & 10,5 \\
Unidades Básicas de Saúde (UBS) Rurais & 1 & 0,5 \\
Clínicas e Comunidades Terapêuticas & 184 & 100,0 \\
Total & &
\end{tabular}

Fonte: Autores

A despeito de 97 serviços prestarem assistência a crianças e adolescentes usuários de drogas, somente quatro abarcam especificamente essa população, sendo nenhum exclusivo sobre drogas: o CAPSi, com demandas de comorbidades psiquiátricas e realizando o cuidado compartilhado com CAPSad e UBS; o CREAS infância e juventude para crianças e adolescentes vítimas de violência ou autores de ato infracional; e duas entidades socioassistenciais não governamentais, sendo uma sobre saúde mental de adolescentes e outra abrangendo demandas sociais e de saúde de crianças e adolescentes. Os quatro serviços estão localizados na região central.

Os serviços com modelos de cuidado abertos foram mais prevalentes do que aqueles com modelos fechados e asilares, que requerem necessariamente um regime de internação. Dos sistemas abertos, destacam-se a abordagem psicossocial através do: CAPSad; dois CAPS gerais, CAPSi, centro de convivência e residências terapêuticas na assistência aos egressos de hospitais psiquiátricos com problemas decorrentes do uso de drogas; no cuidado territorializado das UBS, CRAS, CREAS e equipe de consultório na rua; entidades socioassistenciais; e serviços de acolhimento institucional. Dentro da abordagem psicossocial, grande parte dos serviços engloba o cuidado ambulatorial, como as UBS, todos os CAPS, a equipe de consultório na rua e uma entidade socioassistencial. Contudo, alguns dispositivos, por conta de suas naturezas, restringem-se somente à abordagem ambulatorial, como os ambulatórios em saúde mental e álcool e outras drogas, focados em consultas clínicas individuais. Existem também nesse tipo de sistema os 44 GAMs. 
Com relação aos sistemas fechados/asilares que pressupõem internação, quatro serviços abrangem o tratamento em regime de internação para desintoxicação, sendo: três serviços hospitalares governamentais com leitos e internação de curto prazo, um somente para homens e os outros para ambos os sexos, além do hospital que somente atendia pessoas em situação de encarceramento. Além destes, as 20 clínicas e CTs seguem os preceitos desse modelo residencial de tratamento, com internação de curto, médio a longo prazo, e um hospital psiquiátrico requer internação psiquiátrica de tempo variado.

Referente ao financiamento dos serviços, 122 recebem algum tipo de financiamento público. Desses, 106 eram entidades governamentais e 16 não governamentais, sendo oito CTs, quatro entidades socioassistenciais, três serviços de acolhimento institucional e um hospital psiquiátrico que estava em intervenção municipal para fechamento. Cinquenta e cinco serviços recebem doações de pessoas físicas e/ou jurídicas, duas instituições eram autossustentáveis e os 11 serviços privados são clínicas/CTs. Nove instituições possuem mais de uma forma de financiamento.

Tabela 3. Perfis dos Serviços que Prestam Assistência a Usuários de Drogas no Município

\begin{tabular}{|c|c|c|}
\hline Variáveis & $\mathbf{N}$ & $\%$ \\
\hline \multicolumn{3}{|l|}{ Foco da Assistência } \\
\hline Usuários de drogas & 176 & 95,7 \\
\hline Familiares de usuários de drogas & 8 & 4,3 \\
\hline \multicolumn{3}{|l|}{ Faixa Etária } \\
\hline Crianças e adolescentes (5 a 18 anos) & 97 & 52,7 \\
\hline Adultos (acima de 18 anos) & 181 & 98,4 \\
\hline \multicolumn{3}{|l|}{ Sexo } \\
\hline Feminino & 2 & 1,1 \\
\hline Masculino & 17 & 9,2 \\
\hline Ambos os sexos & 165 & 89,7 \\
\hline \multicolumn{3}{|c|}{$\begin{array}{l}\text { Sistemas de Tratamento de acordo com os modelos } \\
\text { assistenciais }\end{array}$} \\
\hline 1. Sistemas Abertos & 158 & 86,4 \\
\hline 1.1 Psicossocial & 38 & 20,7 \\
\hline 1.2 Ambulatorial & 3 & 1,6 \\
\hline 1.3 Psicossocial/Ambulatorial & 74 & 40,2 \\
\hline 1.4 Ajuda Mútua & 44 & 23,9 \\
\hline 2. Sistemas Fechados/Asilares & 25 & 13,6 \\
\hline 2.1 Internação para Desintoxicação & 4 & 2,2 \\
\hline 2.2 Comunidades Terapêuticas & 20 & 10,9 \\
\hline 2.3 Internação Psiquiátrica & 1 & 0,5 \\
\hline \multicolumn{3}{|l|}{ Financiamento } \\
\hline Próprio & 2 & 1,1 \\
\hline Doações (pessoas físicas e jurídicas) & 55 & 29,9 \\
\hline Público & 122 & 66,3 \\
\hline Privado/Particular (pagamentos dos usuários) & 11 & 6,0 \\
\hline
\end{tabular}

Fonte: Autores

\section{Discussão}

Os resultados apresentados indicam que os serviços de atendimento especializado aos usuários de drogas estão presentes em menor número do que os não-especializados, isto é, os de cunho generalista, o que se mostra coerente com os pressupostos das políticas nacionais, a partir da reformulação do modelo assistencial pela RP (MS, 2004; SENAD, 2005; Portaria $\mathrm{n}^{\circ}$ 3.088, de 23 de dezembro de 2011). Como limitações para uma compreensão aprofundada dos dados acima apresentados, encontram-se a insuficiência de parâmetros nacionais acerca das necessidades e da cobertura assistencial referente às pessoas com problemas relacionados ao uso de drogas, assim como as especificidades socioculturais do contexto brasileiro e do próprio modelo assistencial que dificultam comparações com outros países (Mota, 2016).

Ademais, apesar da heterogeneidade de dispositivos e abordagens, existe uma hegemonia da oferta de serviços não governamentais, principalmente no que se refere aos específicos/especializados na assistência aos usuários de drogas. Essa carência de serviços governamentais é ainda mais acentuada quando se observa que dos seis dispositivos governamentais específicos sobre a temática, somente quatro são abertos à população geral e/ou não restringem sua assistência a nenhum tipo de droga específica. Existem ainda dispositivos governamentais, mas coordenados por entidades não governamentais.

Não se questionam as entidades não governamentais enquanto tentativas da sociedade de abranger a temática, até porque, em um cenário de lacuna assistencial e negligência estatal até o final do século $\mathrm{XX}$, estes esforços se constituíam como umas das parcas formas de cuidado aos usuários de drogas (Alves, 2009). A inclusão dos recursos comunitários de forma complementar também se deve à insuficiência de serviços públicos sobre a temática no Brasil (Carvalho, 2007). Contudo, essa lógica interativa com os recursos comunitários não pressupõe uma super-responsabilização da sociedade na solução do problema, através de uma desresponsabilização do Estado na oferta do cenário de tratamento público. É questionado se a confluência público/privado e o financiamento de entidades não governamentais - já insuficientes como veremos a seguir - em detrimento do financiamento, implantação e gestão de serviços governamentais, não podem resultar no enfraquecimento do SUS, SUAS e políticas 
públicas na área. Nessa direção, cabe ressaltar a existência de intensos e constantes debates acerca desse assunto, sobretudo da inserção das CTs na RAPS e seus financiamentos pela SENAD, contrariando, inclusive, decisões da IV Conferência de Saúde Mental.

Atrelado a isso, uma pesquisa de caracterização dos serviços de atenção à dependência de drogas em Florianópolis observou-se que, dos 28 serviços que prestavam atendimento a usuários de drogas, somente cinco eram governamentais (17,9\%) (Spohr et al., 2006). No Espírito Santo, 250 instituições prestavam tratamento para o uso de drogas, sendo 44 governamentais $(17,6 \%)$, 57 não governamentais (22,8\%) e 149 GAMs $(59,6 \%)$ (Siqueira et al., 2007). Morais (2008) identificou na região centro-oeste 129 instituições de tratamento para usuários de drogas, sendo 85 não governamentais $(65,9 \%)$ e 43 governamentais (33,3\%), com uma não respondente. No levantamento nacional das instituições de atenção a usuários de drogas de 2006 e 2007, apenas 389 instituições de tratamento eram governamentais (31\%), com 867 não governamentais (69\%) (Carvalho, 2007).

A partir da comparação com os dados supracitados, observa-se uma semelhança do panorama assistencial do município com o nacional, demonstrando uma maior prevalência de dispositivos não governamentais para a assistência aos usuários de drogas (Carvalho, 2007). Dessa forma, apesar do caráter local do estudo, algumas generalizações, guardadas as devidas proporções, podem ser realizadas com o contexto nacional, corroborando as críticas que apontam a necessidade de fortalecimento das estratégias públicas e governamentais.

Seguindo as recentes mudanças para a assistência aos usuários de drogas, os sistemas de tratamento abertos, sem a necessidade de internação para o usuário de drogas, apresentam-se em maior número. Estes sistemas abrangentes e contínuos contribuem para a superação da ênfase exclusiva em serviços especializados (Rush, 2010) e para a desmistificação de que o tratamento aos usuários de drogas pressupõe necessariamente a internação (com o isolamento da substância e do convívio social) ou que a internação é a parte final do tratamento (Cetolin, Trzcinski, \& Marchi, 2013; D. R. Schneider \& Lima, 2011; Vinha, 2011).

Este fator, contudo, não exclui a importância dos serviços especializados ou restringe a existência de dispositivos residenciais, que, em consonância com os princípios da RP, podem ser adequados a uma parcela de usuários e determinadas situações. Sabe-se que nem todas as pessoas irão se beneficiar de uma única intervenção ou modelo de tratamento. Destaca-se então a necessidade de se ancorar numa diversificada gama de serviços, ampliando a base de tratamento e continuum de cuidado (Rush, 2010). Como o tratamento residencial no município fica relegado aos dispositivos não governamentais, como as CTs, é premente a implantação de serviços governamentais residenciais, como as Unidades de Acolhimento, aliada a ampliação e descentralização da assistência hospitalar, com internação de curto prazo para desintoxicação e atenção à crise em hospitais gerais (J. F. Schneider et al., 2013).

É importante também a implantação de mais CAPSad e expansão da RAPS no município. Por serem construções recentes, caracterizando uma nova forma de olhar e abordar as pessoas com transtornos mentais e usuários de drogas, devem ser fortalecidos e potencializados de acordo com as características locais. Essa expansão no número de CAPS, que são os principais serviços assistenciais para pessoas com transtornos mentais, e responsáveis também pelo matriciamento de outros serviços e pela articulação da rede, é observada no Brasil. Detecta-se um crescimento de $35,7 \%$ no número de CAPS no país de 2008 a fevereiro de 2014, com 1906 CAPS em 1413 municípios, representando um aumento de 466 municípios com CAPS. Contudo, somente 378 são específicos para usuários de drogas, os CAPSad, com 69 do tipo III funcionando $24 \mathrm{~h}$ por dia (Sala de Apoio à Gestão Estratégica [SAGE], 2014). Essa baixa cobertura é corroborada pelos levantamentos supracitados (Carvalho, 2007; Morais, 2008; Spohr et al., 2006; Siqueira, 2007) e estudos como o de Vinha (2011), que detectou apenas um CAPSad na região de Piracicaba/São Paulo que compreende 25 municípios, bem como a auditoria nacional do Tribunal de Contas da União (TCU) sobre o sistema nacional de políticas sobre drogas (TCU, 2012).

Segundo o parâmetro de cobertura em saúde mental estabelecido pelo MS, que considera adequada a existência de um CAPS para cada 100 mil habitantes, a cobertura dos CAPS em Juiz de Fora gira em torno 0,95 , sendo avaliada como muito boa (acima de 0,70), com o mesmo índice de Minas Gerais $(0,95)$ e acima da média nacional $(0,86)$. É importante ressaltar o crescimento nacional, que possuía um indicador de 0,21 em 2002. (SAGE, 2014). Contudo, em estudo que testou a variabilidade de cobertura de CAPS no Rio Grande do Sul, com um índice de 1,32 - o estado com o segundo maior índice de cobertura do país (SAGE, 2014) -, foram 
observadas sete regiões com cobertura insuficiente/inadequada, representando $49 \%$ da população do estado (Gonçalves et al., 2010). Os autores questionam os parâmetros de cobertura proposto pelo MS, sugerindo análises alternativas por áreas geográficas que identifiquem carências regionais/locais e forneçam subsídios para a extensão da rede de forma igualitária (Gonçalves et al., 2010).

Ademais, é questionado se somente um CAPSad é capaz de suprir as demandas existentes num município de 545.942 habitantes. Ademais, questiona-se a utilização deste parâmetro para saúde mental em geral e álcool e outras drogas, desconsiderando as especificidades de cada área. Se as propostas de tratamento são territorializadas/comunitárias e abertas à sociedade, como pressupõem os CAPS e CAPSad, estes, ao estarem em número insuficientes e centralizados no município, são restringidos de atuarem dessa forma ampliada. Quando uma pessoa precisa sair de seu bairro para frequentar o serviço em outra região, a proposta comunitária fica prejudicada. Essa insuficiência dos serviços também pode obstaculizar o trabalho compartilhado com outros dispositivos e setores, a realização do matriciamento aos serviços não especializados, como UBS e CRAS, e o controle do fluxo de usuários na rede (Fodra \& Rosa, 2009). A insuficiência do matriciamento poderia ser minimizada pelas equipes de NASF, mas que não existiam no município.

Nesse cenário, enfatiza-se a importância da Atenção Básica ( $A B$ ) e Estratégia de Saúde da Família (ESF), que passam a fazer parte da RAPS em 2011, especialmente no provimento de um cuidado territorializado e comunitário abarcando os determinantes sociais. Contudo, a cobertura populacional da $A B$ no município, referente às 67 UBS existentes é de $60,64 \%$, menor que índices nacionais (68,78\%) e estaduais (79,42\%). A cobertura da população pela ESF no município $(55,18 \%)$ também apresenta-se abaixo das porcentagens nacionais $(55,78 \%)$ e estaduais $(70,86 \%)$ (SAGE, 2014). Conforme postula a RAPS, as UBS e equipes de ESF também podem atuar nas ações de promoção à saúde, prevenção, acolhimento, classificação de risco e ordenamento do cuidado compartilhado, a partir de um prisma sociocultural e territorializado (Portaria $\mathrm{n}^{\circ}$ 3.088 , de 23 de dezembro de 2011). Entretanto, a insuficiência de cobertura destes serviços, vinculada a outros fatores, como estigmas e déficit de formação/qualificação para lidar com a temática, insuficiência estruturais (D. R. Schneider \& Lima, 2011; Silveira \& Ronzani, 2011), pode ocasionar em uma alta demanda e sobrecarga profissional, restringindo a atuação das UBS e ESF ao encaminhamento aos serviços especializados (Araújo, 2013; Costa et al., 2015; J. F. Schneider et al. 2013).

Assim, em consonância com Costa et al. (2015), por mais que a $A B$ desponte como o principal nível de atenção nos discursos oficiais e documentos políticos, deve-se refletir sobre como tais predicados não reverberam necessariamente em sua potencialização na prática, acarretando em uma série de limitações para sua atuação, o que por sua vez, também não significa desconsiderá-la deste panorama. Tais ponderações são importantes para que não caiamos em processos de culpabilização e manutenção de uma lógica de transferência de responsabilidades, frequentemente sob a alcunha de descentralização, que relegam à $A B$ e seus profissionais, muitas das vezes, responsabilidades aquém de sua capacidade e função, num cenário de sucateamento e subfinanciamento (Costa et al., 2015).

Faz-se necessária também a ampliação da rede de urgência/emergência e nível hospitalar para usuário de drogas. O Serviço de Urgência Psiquiátrica do Hospital de Pronto Socorro e o SAMU são responsáveis por toda demanda de urgência/emergência da rede pública da área. Também é importante a conformação de mais leitos hospitalares para desintoxicação (existiam somente 24 em um hospital geral), salas de estabilização e outros dispositivos para usuários de drogas, visando desobstruir estes níveis de atenção. Especificamente sobre o hospital do sistema prisional, é importante frisar que este foi inserido no estudo, como parte da rede de atenção aos usuários de drogas, por prestar assistência a usuários de drogas, atendendo também aos outros pré-requisitos elencados no método. Entretanto, tem-se ciência das contradições inerentes a seus modelos, estatutos e práticas vigentes no âmbito da saúde mental, cujo caráter predominantemente custodial apresenta uma série de dissonâncias frente aos postulados da RP (Cordioli, Borenstein, \& Ribeiro, 2006).

Cabe ressaltar que todo este cenário de insuficiência não se restringe ao município estudado, mas é fruto do projeto neoliberal que orienta a concepção sobre o Estado e a condução das políticas públicas e que no Brasil ganha terreno e força a partir dos anos 1990. Consequentemente, tem-se a desregulamentação e desresponsabilização estatal e a responsabilização da chamada sociedade civil, ancorada à mercantilização dos direitos e serviços conformando uma série de desafios para a implantação da RP e à abordagem ampliada 
e contextualizada sobre a temática do uso de drogas, dentre outros fenômenos que perpassam a vida dos brasileiros e, ao fazerem parte da totalidade social, a conformam e são conformados por ela. Como implicações para a área da saúde mental e álcool e outras drogas, observa-se seu sucateamento e subfinanciamento, com terceirização e privatização de serviços e de sua gestão e precarização das condições e vínculos de trabalho, retroalimentando os antagonismos sociais e pauperização das condições de vida materiais e subjetivas da população (Vasconcelos, 2012).

A partir dos dados apresentados, discute-se também a atenção a grupos populacionais específicos, como crianças e adolescentes e mulheres. Apesar desses grupos populacionais não constituírem a maioria das prevalências e perfis de usuários e dependentes no Brasil, os seguintes aspectos devem ser considerados: 1 ) maior suscetibilidade das mulheres ao sexo/prostituição para manutenção da dependência, com maior chance de sofrerem violência sexual, engravidarem ou contraírem doenças sexualmente transmissíveis; 2) consequências do uso de drogas na infância e adolescência para o seu desenvolvimento cognitivo e pessoal, dentre outros pontos importantes (Carlini et al., 2007; Kessler et al., 2003; Oliveira, Nascimento, \& Paiva, 2007). Ademais, a própria literatura científica demonstra que adolescentes e mulheres têm mais dificuldades em aderir e prosseguir com o tratamento (Kessler et al., 2003; Silva et al., 2003). Além do estigma associado ao usuário de drogas (Silveira \& Ronzani, 2011), a predominância de serviços dirigida a um perfil masculino de idade adulta, ou que englobam várias faixas etárias e todos os sexos de forma homogênea, pode fazer com que pessoas e/ou grupos populacionais que não estejam nessas padronizações tenham dificuldade de aderir ao tratamento (Silva et al., 2003; Spohr et al., 2006).

Problematiza-se, assim, a identificação de somente quatro serviços especializados para crianças e adolescentes, sendo nenhum deles específico sobre drogas, e dois serviços exclusivos para o tratamento de mulheres, com o único sobre drogas sendo particular. Estas insuficiências assistenciais também são corroboradas pelos levantamentos anteriores (Carvalho, 2007; Morais, 2008; Siqueira, 2007 Spohr et al., 2006). Uma limitação do estudo é a divisão de faixas etárias entre crianças/adolescentes e adultos, não considerando idosos e diversos aspectos sociais.

Por fim, constata-se as desigualdades de cobertura entre as áreas urbana e rural, cuja avaliação é limitada pelas lacunas existentes à compreensão das condições de vida e acessos às políticas públicas da população rural, proveniente de descasos tanto das próprias políticas quanto da academia (Dimenstein, Leite, Macedo, \& Dantas, 2016). Assim, conforme Ronzani, Costa e Paiva (2016), as políticas da área homogeneízam o território e realidades vivenciadas, aos não distinguirem as regiões urbanas e rurais, desconsiderando as múltiplas especificidades existentes e relegando aos moradores de áreas rurais a assistência prestada por equipes da $A B$ fixas ou móveis e o deslocamento aos bairros urbanos para serem atendidos.

Outras limitações enfrentadas pelo estudo são: indisponibilidade de indicadores sociais das regiões; a estratégia de bola de neve, gerando possíveis desconsiderações de serviços; e ausência de outras especificações sobre os serviços e assistência oferecida, capacitação dos profissionais, assim como indicadores de desfecho. Contudo, acredita-se que estas limitações não invalidam os achados e as discussões suscitadas, devido à própria relevância da temática e também à forma como é abordada, a partir de um levantamento que concebe a rede de atenção aos usuários de drogas de forma ampliada e intersetorial, não focando somente nos serviços especializados e da saúde.

\section{Conclusões}

Os seguintes pontos da rede de atenção aos usuários de drogas analisada são destacados: necessidade de ampliação da rede de AB; implantação de mais CAPSad; expansão da RAPS; reestruturação da atenção a grupos populacionais, como mulheres, crianças e adolescentes; ampliação da rede de urgência e emergência; e maior distribuição dos serviços, principalmente os governamentais especializados. Cabe ressaltar que estes aspectos também são observados em outras pesquisas sobre a assistência aos usuários de drogas no Brasil, indicando caminhos para as políticas públicas na área.

Estudos futuros enfocando a avaliação de necessidades dos usuários de drogas podem potencializar os achados de pesquisas como essa, voltadas para o levantamento das redes de atenção, ao extraírem taxas de prevalência e incidência e estimativas populacionais das necessidades de saúde dos usuários a serem atendidas pela rede. Ademais, são necessárias novas pesquisas buscando entender de forma ampliada outras realidades assistenciais aos usuários de drogas do país, aprofundando a compreensão destas redes e abarcando 
os aspectos relacionais dos serviços e pessoas. Dessa forma, o presente levantamento apresenta uma série de potencialidades e possibilidades de continuidade para pesquisas e análises futuras da rede, tais como: (a) a possibilidade de aprofundamento dos achados a partir de delineamentos observacionais; (b) a utilização da Análise de Redes Sociais, identificando e avaliando as relações estabelecidas entre os serviços e abrangendo o dinamismo das redes de atenção aos usuários de drogas; (c) análises que levem em conta indicadores sociais e estruturais do município, bairros e regiões, compreendendo a importância dos contextos.

\section{Referências}

Alves, V. S. (2009). Modelos de atenção à saúde de usuários de álcool e outras drogas: discursos políticos, saberes e práticas. Cadernos de Saúde Pública, 25(11), 2309-19. doi: 10.1590/ S0102-311X2009001100002

Araújo, A. C. C. (2013). Atenção Primária e dependência química: contribuições do matriciamento em saúde mental. Saúde em Debate, 37(spe), 61-9. Recuperado de http://cebes.org.br/site/wp-content/ uploads/2014/06/RSD_EspecialDrogas_REV2905_Web.pdf

Biernacki, P., \& Waldford, D. (1981). Snowball sampling: problems and techniques of chain referral sampling. Sociological Methods \& Research, 10(2), 141-63. doi: 10.1177/004912418101000205

Carlini, E. A., Galduróz, J. C. F., Noto, A. R., Carlini, C. M. A., Oliveira, L. G., Nappo, S. A., ... Sanchez, Z. V. D. (2007). II Levantamento domiciliar sobre o uso de drogas psicotrópicas no Brasil: estudo envolvendo as 108 maiores cidades do país - 2005. Brasília, DF: Secretaria Nacional Antidrogas.

Carvalho, D. B. B., coordenador. (2007). Mapeamento das instituições governamentais e não governamentais de atenção às questões relacionadas ao consumo de álcool e outras drogas no Brasil - 2006/2007. Relatório. Brasília, DF: Secretaria Nacional Antidrogas.

Cetolin, F. S., Trzcinski, C., \& Marchi, A. C. W. (2013). A internação de usuários de álcool e outras drogas em Hospital Geral. Saúde em Debate, 37(spe), 122-9. Recuperado de http://cebes.org.br/site/ wp-content/uploads/2014/06/RSD_EspecialDrogas_REV2905_ Web.pdf

Cordioli, M. S., Borenstein, M. S., \& Ribeiro, A. A. A. (2006). Hospital de custódia: os direitos preconizados pela reforma psiquiátrica e a realidade dos internos. Escola Anna Nery, 10(4), 671-677. doi: 10.1590/S1414-81452006000400008

Costa, P. H. A., Mota, D. C. B., Paiva, F. S., \& Ronzani, T. M. (2015). Desatando a trama das redes assistenciais sobre drogas: uma revisão narrativa da literatura. Ciência \& Saúde Coletiva, 20(2), 395406. doi: 10.1590/1413-81232015202.20682013

Dimenstein, M. D., Leite, J., Macedo, J. P., \& Dantas, C. (Orgs.). (2016). Condições de vida e saúde mental em contextos rurais. São Paulo: Intermeios

Fodra, R. E. P., \& Rosa, A. C. (2009). Centro de Atenção Psicossocial Álcool e Drogas (CAPS ad): análises dos discursos e da prática no contexto da Reforma Psiquiátrica e atenção psicossocial. Saúde em Debate, 33(81), 129-9.
Gonçalves, V. M., Abreu, P. S., Candiago, R. H., Saraiva, S. S., Lobato, M. I., \& Belmonte-de-Abreu, P. S. (2010). A falácia da adequação da cobertura dos Centros de Atenção Psicossocial no estado do Rio Grande do Sul. Revista de Psiquiatria do Rio Grande do Sul, 32, 16-8. doi: 10.1590/S0101-81082010000100003

Instituto Brasileiro de Geografia e Estatística. (2014). Cidades@. Recuperado de http://cidades.ibge.gov.br/painel/painel. php?codmun=313670.

Kessler, F., von Diemen, L., Seganfredo, A. C., Brandão, I., Saibro, P., Scheidt, B., ... Ramos, S. P. (2003). Psicodinâmica do adolescente envolvido com drogas. Revista de Psiquiatria do Rio Grande do Sul, 25(1), 33-41. doi: 10.1590/S0101-81082003000400005

Ministério da Saúde (2004). A Política do Ministério da Saúde para Atenção Integral a Usuários de Álcool e outras Drogas. Brasília, DF: Ministério da Saúde.

Morais, R. P. (2008). Redes socioassistenciais de tratamento aos usuários/dependentes de álcool e outras drogas na região CentroOeste (Brasil 2006/2007) (Monografia de conclusão de Curso). Universidade de Brasília. Recuperado de http://bdm.unb.br/bitstream/10483/762/1/2008_RozaniaPiresMorais.pdf

Mota, D. C. B. (2016). Rede assistencial para usuários de álcool e outras drogas: desenvolvimento de um modelo de planejamento baseado em necessidades (Tese de Doutorado não publicada). Universidade Federal de Juiz de Fora.

Oliveira, J. F., Nascimento, E. R., \& Paiva, M. S. (2007). Especificidades de usuários(as) de drogas visando uma assistência baseada na heterogeneidade. Escola Anna Nery, 11(4), 694-8. doi: 10.1590/ S1414-81452007000400022

Portaria no 3.088. (2011, 23 de dezembro). Institui a Rede de Atenção Psicossocial para pessoas com sofrimento ou transtorno mental e com necessidades decorrentes do uso de crack, álcool e outras drogas, no âmbito do Sistema Único de Saúde. Brasília, DF: Ministério da Saúde.

Ronzani, T. M., Costa, P. H. A., \& Paiva, F. S. (2016). Fatores associados ao uso de álcool em assentamentos rurais. In M. D. Dimenstein, J. Leite, J. P. Macedo, \& C. Dantas (Orgs.), Condições de vida e saúde mental em contextos rurais (pp. 281-296). São Paulo: Intermeios.

Rush, B. (2010). Tiered frameworks for planning substance use service delivery systems: origins and key principles. Nordic Studies on Alcohol and Drugs, 27(6), 617-36. Recuperado de http://journals. sagepub.com/doi/pdf/10.1177/145507251002700607

Sala de Apoio à Gestão Estratégica [SAGE]. (2014). Recuperado de http://189.28.128.178/sage/.

Schneider, D. R., \& Lima, D. S. (2011). Implicações dos modelos de atenção à dependência de álcool e outras drogas na rede básica em saúde. Psico, 42(2), 168-178.

Schneider, J. F., Roos, C. M., Olschowsky, A., Pinho, L. B., Camatta, M. W., \& Wetzel, C. (2013). Atendimento a usuários de drogas na perspectiva dos profissionais da estratégia saúde da família. Texto\& Contexto - Enfermagem, 22(3): 654-61. doi: 10.1590/ S0104-07072013000300011

Secretaria Nacional de Políticas sobre Drogas (2005). Política Nacional sobre Drogas. Brasília, DF: SENAD.

Silva, V. A., Aguiar, A. S., Felix, F., Rebello, G. P., Andrade, R. C., \& Mattos, H. F. (2003). Brazilian study on substance misuse in adolescents: associated factors and adherence to treatment. Revista Brasileira de Psiquiatria, 25(3), 133-8. doi: 10.1590/ S1516-44462003000300004 
Silveira, P. S., \& Ronzani, T. M. (2011). Estigma social e saúde mental: quais as implicações e importância do profissional de saúde? Revista Brasileira de Saúde da Família, 28, 51-8.

Siqueira, M. M., Barbosa, D. A., Laranjeira, R., \& Hopkins, K. (2007). Psychoactive substances and the provision of specialized care: the case of Espirito Santo. Revista Brasileira de Psiquiatria, 29(4), 31523. doi: $10.1590 / S 1516-44462006005000043$

Spohr, B., Leitão, C., \& Schneider, D. R. (2006). Caracterização dos serviços de atenção à dependência de álcool e outras drogas na região da Grande Florianópolis. Revista de Ciências Humanas, 39, 219-36. Recuperado de https://periodicos.ufsc.br/index.php/ revistacfh/article/view/17997
Tribunal de Contas da União (2012). Sistema nacional de políticas públicas sobre drogas. Brasília, DF: Tribunal de Contas da União.

Vasconcelos, E. M. (2012). Crise mundial, conjuntura política e social no Brasil, e os novos impasses teóricos na análise da reforma psiquiátrica no país. Cadernos Brasileiros de Saúde Mental, 4(8), 8-21. Recuperado de http://incubadora.periodicos.ufsc.br/index.php/ cbsm/article/view/2033/2331

Vinha, I. R. (2011). Cenário da assistência em saúde mental/uso de substâncias psicoativas na região de saúde de Piracicaba, São Paulo, Brasil. SMAD, Revista Eletrônica Saúde Mental Álcool e Drogas, 7(1), 25-31. doi: 10.11606/issn.1806-6976.v7i1p25-31

Pedro Henrique Antunes da Costa, Mestre em Psicologia pela Universidade Federal de Juiz de Fora (UFJF), é Doutorando em

Psicologia pela Universidade Federal de Juiz de Fora (UFJF) e Professor do curso de Psicologia da Faculdade Machado Sobrinho

(FMS) de Juiz de Fora. Endereço para correspondência: Rua José Lourenço, 393. 28/503. CEP: 36036-630, São Pedro, Juiz de Fora. Telefones: (32) 3031-4880 e (32) 98869-0880. E-mail: phantunes.costa@gmail.com

Amata Xavier Medeiros, Graduada em Psicologia pela Universidade Federal de Juiz de Fora (UFJF), é Mestranda em Psicologia pela Universidade Federal de Juiz de Fora (UFJF).

E-mail: amata.medeiros@gmail.com

Bárbara Pereira Loures, Graduada em Psicologia pela Universidade Federal de Juiz de Fora (UFJF), é Trainee na empresa Duratex S.A.

E-mail: barbara.loures@gmail.com

Wanderson Maurício Duarte Silva, Graduando em Psicologia pela Universidade Federal de Juiz de Fora (UFJF).

E-mail: wandersonmduarte@gmail.com

Telmo Mota Ronzani, Doutorado em Ciências da Saúde pela Universidade Federal de São Paulo (UNIFESP) e Pós-doutorado pela Universidade de São Paulo (USP) com estágio Pós-doutoral pela University of Connecticut Health Center (UCHC), é Professor do Departamento e Programa de Pós-graduação em Psicologia da Universidade Federal de Juiz de Fora (UFJF). E-mail: tm.ronzani@gmail.com

Fernando Antonio Basile Colugnati, Doutorado em Ciências pela Universidade Federal de São Paulo (UNIFESP) e Pós-doutorado na

Universidade Estadual de Campinas (UNICAMP), é Professor da Faculdade de Medicina e dos Programa de Pós-graduação Psicologia e da Saúde na Universidade Federal de Juiz de Fora (UFJF). E-mail: fcolugnati@gmail.com

Recebido em 29.Set.15 Revisado em 03.Ago.16 Aceito em 02.Jun.17 